\title{
PARTICLE-IN-CELL SIMULATIONS OF LOWER-DENSITY CM-SCALE CAPILLARY CHANNELS*
}

\author{
P. Messmer ${ }^{\dagger}$, D. Bruhwiler, D. Dimitrov, P. Stoltz, Tech-X Corporation, Boulder, CO 80303, USA \\ W.P. Leemans, E. Esarey, C. Geddes, LBNL, Berkeley, CA 94720, USA
}

\begin{abstract}
Capillary channels of cm-length and at plasma density low compared to gas jets are promising setups for low noise laser wakefield acceleration. Computationally, however, the large discrepancy of the length scales of the plasma and the laser are a big challenge. Methods are therefore sought that relax the need to concurrently resolve both length scales. Average methods, which split the electromagnetic field into a fast and a slowly varying part, allow to relax the constraint to resolve the laser wavelength. Such an envelope model is currently being incorporated into the VORPAL plasma simulation code. Simulation results for benchmark cases and for laser pulse propagation in a $\mathrm{cm}$ scale channel are presented.
\end{abstract}

\section{INTRODUCTION}

The maximum achievable accelerating gradient is orders of magnitude larger when sustained by the collective fields of a high-density plasma, rather than an evacuated metal structure. This has been clearly demonstrated for electrons in laser wakefield accelerator (LWFA) experiments $[1,2,3]$. Plasma-based electron acceleration concepts (see [4] for a review) can sustain longitudinal electric fields of the order of the non-relativistic wavebreaking field, $E_{0}=c m_{e} \omega_{p} / e$, where $\omega_{p}^{2}=4 \pi n_{e} e^{2} / m^{2}$ is the plasma frequency at an electron density $n_{e}$. For $n_{e}=10^{18} \mathrm{~cm}^{-3}$, the electric field is $E_{0} \approx 100 \mathrm{GV} / \mathrm{m}$, with a phase velocity close to the speed of light.

Laser pulse propagation in vacuum is limited by diffraction to the Rayleigh length, $z_{0}=\pi w_{0}^{2} / \lambda$, where $w_{0}$ is the waist size and $\lambda$ the wavelength. In order to sustain the interaction between these strong wakefields and the accelerating electrons, the laser pulse must be guided over many Rayleigh lengths (see e.g. [5] and references therein). For example, the well-defined electron beams seen in [1], comparable in charge and energy to those of [2,3], where obtained with much less laser energy because the lower energy laser pulse was guided by a plasma channel.

Ongoing work at the L'OASIS facility of LBNL, directed toward the production of $\mathrm{GeV}$ electron beams from a LWFA, now includes the propagation of $100 \mathrm{TW}$ scale laser pulses down multi-cm long plasma channels, generated from $10^{18} \mathrm{~cm}^{-3}$ hydrogen gas in a capillary discharge [6]. For such plasma densities (and lower), the use

\footnotetext{
* Work supported by DOE contracts DE-FC02-01ER41178, DE-FG0204ER84097, DE-AC03-76SF00098 and DE-FG03-95ER40926, including the SciDAC Accelerator Project and use of NERSC.

† messmer@txcorp.com
}

of Ti-Sapphire laser technology with $\lambda=0.8 \mu \mathrm{m}$ leads to a very high ratio of $\omega / \omega_{p}$, making it computationally extremely difficult to simultaneously resolve the small laser wavelength, while capturing the larger spatial scales and long propagation times. For these reasons, a ponderomotive treatment of the laser pulse is essential for modeling LWFA experiments such as those now underway at LBNL.

\section{ENVELOPE MODEL}

Ponderomotive guiding center models $[7,8]$ decompose the electromagnetic field in a rapidly varying laser component and the slowly varying wake. The laser envelope is advanced in a semi-analytic way, leaving only the slow time scales to be simulated. This can lead to a substantial saving in computing time, as only the slow timescales have to be resolved. We therefore implemented a model similar to the one in by Gordon et al. [7] in the versatile plasma simulation code VORPAL [9].

The main difference to the model in [7], where the wake vector potential was obtained by solving Poisson's equation, is that we evolve the electric and magnetic field of the wake by using the electromagnetic PIC algorithm with charge conserving current deposition.

Accurately up to order $1 / \omega^{2}$, the laser pulse envelope is evolved in the laboratory frame by solving

$$
\left(c \partial_{x}+\partial_{t}\right) a=\frac{c^{2}}{2 i \omega}\left(1+\frac{c}{i \omega} \partial_{x}\right)\left(\chi+\Delta_{\perp}\right) a
$$

where $a$ is the complex laser pulse envelope, $c$ is the speed of light, $\omega$ is the laser frequency, $\Delta_{\perp}$ is the transverse Laplacian and $\chi=-\mu_{0} \sum \frac{q_{i} \rho_{i}}{\left\langle m_{i}\right\rangle}$ is the coupling parameter to the plasma, with $q_{i}, \rho_{i},\left\langle m_{i}\right\rangle$ the charge, charge density and the relativistic mass of particle $i$. This equation can be solved using a Crank-Nicholson scheme, resulting in a sparse system of linear equations. We use an iterative Krylov subspace solver from the parallel solver package AZTEC [10], which is already used in VORPAL for the parallel electrostatic solver [11]. This approach ensures that the envelope model can be enhanced relatively easy, e.g. to treat the $1 / \omega^{2}$ terms correctly. One problem associated with the AZTEC package is that it only provides solvers for real quantities. The linear system representing Eq. 1 therefore has to treat the real and imaginary part of the envelope separately.

The particle-push algorithm includes the ponderomotive force in addtion to the Newton-Lorentz force. The acceleration is determined by an implicit update of the relativistic 
mass, $\left\langle m_{i}\right\rangle$,

$$
\left\langle m_{i}^{t}\right\rangle=\left\langle m_{i}^{t-1 / 2}\right\rangle+q\left(E-\frac{q}{4\left\langle m_{i}^{t}\right\rangle} F^{t}\right) \frac{P^{t-1 / 2}}{\left\langle m^{t-1 / 2}\right\rangle c^{2}} \frac{d t}{2}
$$

followed by a Boris push in the magnetic field. Finally, the particle position is updated by using

$$
x^{t+1}=x^{t}+\frac{P^{t+1 / 2}}{\left\langle m_{i}^{t-1 / 2}\right\rangle} d t
$$

This final step requires knowledge of $\left\langle m_{i}^{t+1 / 2}\right\rangle$ at position $x^{t+1 / 2}$. Gordon et al used a Taylor expansion around $x^{t}$ in order to determine $\left\langle m^{t+1 / 2}\right\rangle$. But unlike in [7] we were not able to use the quadratic formula to solve this cubic equation. Alternatively we solved it by performing a few iterations of a Newton scheme.

\section{BENCHMARK TEST}

In order to gain confidence in our implementation, we performed a variety of benchmark tests. In the following sections, we present some of the test results.

\section{Vacuum propagation}

A first benchmark to test the envelope model is to investigate laser pulse propagation in vacuum. Figure 1 shows amplitude contours for a laser pulse launched at $z=-3 z_{0}$, where $z_{0}=k w_{0}^{2} / 2$ is the Rayleigh length, $k$ the laser pulse wave number and $w_{0}$ the pulse width. The size of the computational domain is $12 z_{0} \times 20 w_{0}$, discretized on a $70 \times 60$ cell grid.

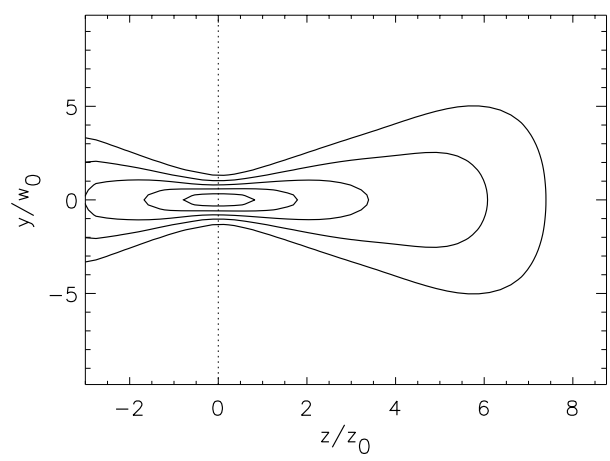

Figure 1: Amplitude contour plot of a laser pulse launched into a vacuum domain. The dotted line represents the focus.

A measure for the accuracy of the envelope evolution is the phase shift experienced by the laser pulse when propagating from $z=-z_{0}$ to $+z_{0}$. Figure 2 compares the phase shift observed in the simulation for different transverse resolutions. The simulation parameters are identical to the previous example. For a transverse resolution of $w_{0} / \Delta y=4$, a good agreement with the theoretical phase shift can be found. At a resolution of $w_{0} / \Delta y=2$, a phase error of about $5 \%$ can be observed.

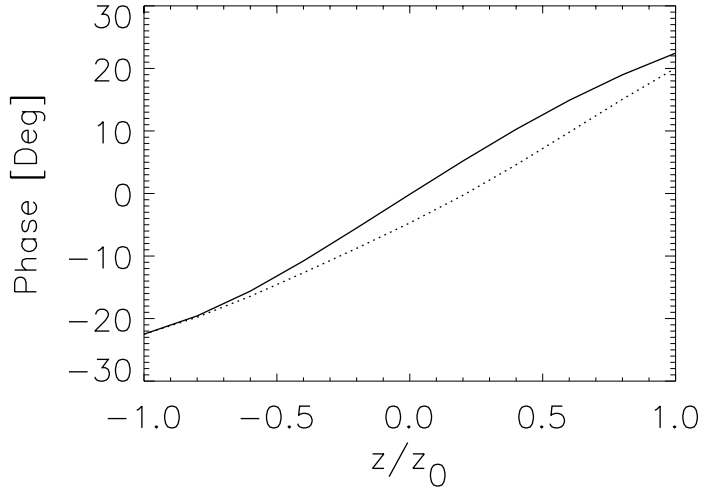

Figure 2: Envelope phase during propagation through the focus for transverse resolutions of the laser pulse of $w_{0} / \Delta y=4$ (solid) and $w_{0} / \Delta y=2$ (dotted). The theoretical phase shift is 45 degree (Guoy Phase shift).

\section{Comparison to PIC}

The tests in the previous section demonstrated the correct working of the envelope evolution in vacuum. The next test is now to ensure the correct interaction of the pulse envelope with a plasma.

Therefore, a laser pulse with normalized amplitude $a_{0}=$ 1 was injected into a plasma of density $n=1.74 \cdot 10^{19} \mathrm{~cm}^{-3}$ with a ratio of laser frequency to plasma frequency $\omega / \omega_{p}=$ 10. The plasma density is ramped up linearly over a distance of $30 \mu \mathrm{m}$. The simulation was performed on a domain of $40 \times 64 \mu \mathrm{m}$. In case of the PIC simulation, the domain was discretized on a $800 \times 1280$ cell grid. This fine resolution is necessary to resolve the laser wavelength. The timestep is $1.12 \cdot 10^{-16} \mathrm{~s}$, determined by the CFL condition.

Using the envelope model, the same computational domain can be captured with a much smaller computational grid. The above system can be modeled on a $80 \times 128$ cell grid with a timestep of $1.12 \cdot 10^{-15} \mathrm{~s}$. The grid is 100 times smaller and the time step 10 times larger than for the full PIC model. Note that the timestep in the envelope model is still bound by the CFL condition, as the wake field is updated using an explicit integration of a finite differenced version of Maxwell's equations. But the coarser grid, which now no longer needs to resolve the laser wave length, allows a much larger timestep.

Figure 3 shows the longitudinal wake electric field after the laser pulse has penetrated the plasma. The envelope model captures all the features of the wake quantitatively, at a considerably lower cost: In both PIC and envelope model, 5 particles/cell were used on average. The computational time for the PIC model was approximately 200 times larger than for the envelope model.

Another test is to investigate the response of a plasma on laser pulses with different peak electric fields. Figure 4 shows the wake electric field for a non-relativistic normalized amplitude of $a_{0}=0.6$ and for a relativistic case with 


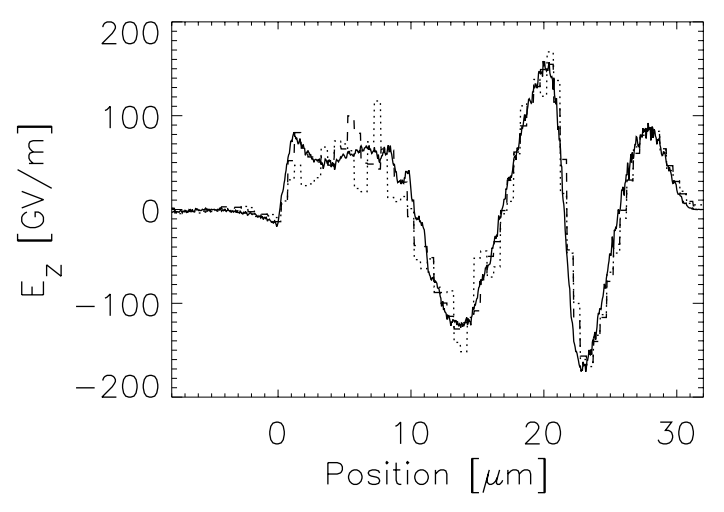

Figure 3: Longitudinal electric field in the wake of a laser pulse, computed with full PIC (solid), the envelope model on a 100 times smaller grid and 10 times less particles (dotted) and for the same grid size with 100 times less particles (dashed). The plasma extends between $x=0$ and $x=32 \mu m$ with a linear density ramp in the first $20 \mu \mathrm{m}$.

$a_{0}=2.5$. In the non-relativistic case, a sinusoidal wake is formed behind the laser pulse. In the relativistic case, non-linear wave steepening can be observed.

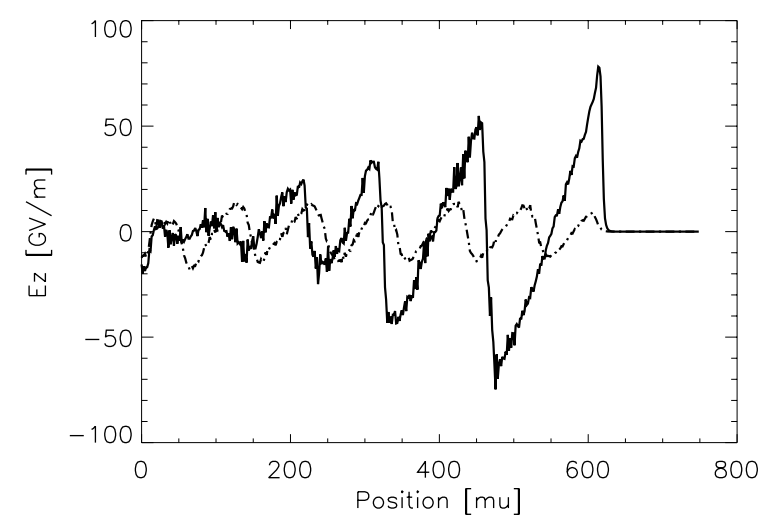

Figure 4: Comparison of the wake behind a laser pulse for $a_{0}=2.5$ (solid) and $a_{0}=0.6$ (dotted).

\section{Channel simulation}

In order to check the benefit from a channel, we performed a simulation of a laser pulse propagating in a preformed channel of $0.8 \mathrm{~cm}$ length, using the newly developed envelope model. The density at the channel bottom is $1.25 \cdot 10^{17} \mathrm{~cm}^{-3}$, corresponding to a plasma wave length of $L=2 \pi c / \omega_{p}=95 \mu \mathrm{m}$. The channel has a parabolic profile with opening $20 \mu \mathrm{m}$. A matched $3.17 \cdot 10^{-13} s$ laser pulse with $\omega=2.35 \cdot 10^{15} \mathrm{~s}^{-1}$, a Rayleigh length $z_{0}=0.157 \mathrm{~cm}$ and amplitude $a_{0}=1.0$ is sent into the channel. The simulation captures a domain of $0.9 \mathrm{~cm} \times 120 \mu \mathrm{m}$, discretized on a $3000 \times 40$ cell grid.

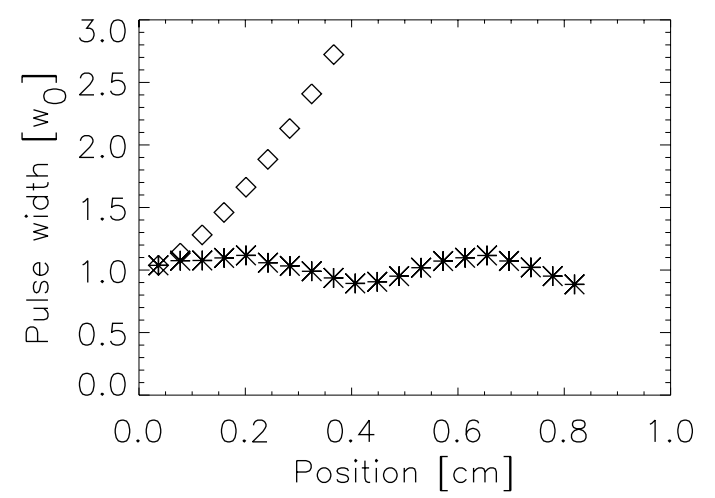

Figure 5: Laser pulse width for propagation in a channel (stars) and without a channel (diamonds).

Figure 5 shows the width of the laser pulse while it propagates through the plasma channel. For comparison, the evolution of the pulse without a channel is shown. Obviously, the laser pulse is guided in the channel without significantly changing its width.

\section{Conclusion}

Using a ponderomotive guiding center algorithm as implemented in VORPAL allows to model large scale systems for laser-plasma interaction. Especially at low plasma densities significant saving in computing time can result. It has been demonstrated that the model can reproduce both analytical and PIC simulation results and that it can be used to model laser pulse propagation in a low density channel.

\section{REFERENCES}

[1] C.G.R.Geddes, C. Toth, J. van Tilborg, E. Esarey, C.B. Schroeder, D. Bruhwiler, C. Nieter, J.R.Cary, and W.P. Leemans, Nature, 431, 538, 2004.

[2] S.P.D. Mangles, et al., Nature, 431, 535, 2004.

[3] J. Faure, et al., Nature, 431, 541, 2004.

[4] E. Esarey, et al, IEEE Trans. Plasma Science, 24, 252, 1996

[5] E. Esarey, W.P. Leemans, Proc. Part. Accel. Conf, 3699, 1999.

[6] W.P. Leemans, et. al., TPAE019, this proceedings.

[7] D.F. Gordon, et al., IEEE Transactions on Plasma Sci, 28 (4), $1135,2000$.

[8] P. Mora, T.M. Antonsen, Phys. Plasmas, 4, 217, 1997.

[9] C. Nieter, J.R. Cary, J. Comp. Phys., 196, 448, 2004.

[10] R.S. Tuminaro, et al., SAND99-8801J, 1999.

[11] P. Messmer, D.L. Bruhwiler, Comp. Phys. Comm., 164, 118, 2004. 